\title{
EFEITOS DO GRUPO GENÉTICO, SEXO E PESO AO ABATE SOBRE AS PROPRIEDADES FÍSICO-QUIMMICAS DA CARNE DE CORDEIROS EM CRESCIMENTO'
}

\author{
Xisto Rodrigues SOUZA², Maria Cristina BRESSAN²,*, Juan Ramón Olalquiaga PÉREZ³, \\ Peter Bitencourt FARIA, Josye Oliveira VIEIRA, Deyse Marilda KABEYA
}

\section{RESUMO}

O objetivo desse trabalho foi avaliar a evolução do pH post mortem, cor, perda de peso por cozimento e força de cisalhamento dos músculos Longissimus dorsi (LD) e Semimembranosus (SM) de 49 cordeiros provenientes de cruzamentos entre as raças Bergamácia com Santa Inês (BgxSI) e Ile de France com Santa Inês (IFxSI), machos inteiros e fêmeas, distribuídos nos grupos pesos ao abate de 15, 25, 35 e $45 \mathrm{~kg}$. Os grupos genéticos apresentaram diferenças $(\mathrm{P} \leq 0,05)$ sobre o $\mathrm{pH}$ dos músculos LD e SM, sendo que BgxSI apresentou média de $\mathrm{pH}$ mais elevada, do que IFxSI. O fator sexo não afetou a cor, entretanto o grupo genético influenciou $(\mathrm{P}<0,001)$ o valor $\mathrm{L}^{*}$ e o valor a*; o valor L* de 35,25 e 32,89, para IFxSI e BgxSI, respectivamente; e o valor a* de 16,09 e 14,64, para BgxSI e IFxSI, respectivamente. A perda de peso por cozimento não foi influenciada pelos fatores grupos genéticos, sexos e grupos de peso. A maciez no músculo SM não foi afetada pelos fatores estudados. Entretanto no LD, os grupos de peso afetaram a maciez $(\mathrm{P}<0,05)$. Os grupos de 15 e $25 \mathrm{~kg}$ apresentaram força de cisalhamento mais elevada (13,57 e 10,98kgf, respectivamente), do que os cordeiros de 35 e $45 \mathrm{~kg}(8,56$ e $7,97 \mathrm{kgf}$, respectivamente).

Palavras-chave: ovinos; qualidade de carne; maciez.

\section{SUMMARY}

EFFECTS OF BREED GROUP, GENDER AND SLAUGHTER WEIGHT GROUP ON THE PHYSICAL-CHEMICAL PARAMETER OF THE GROWING LAMBS. The objective of this work was to evaluate the post mortem pH evolution, color, cooking losses and shear force of longissimus dorsi (LD) and semimembranosus (SM) muscles of forty nine sheep from two breed group, Bergamácia x Santa Inês (BgxSI) and Ile de France x Santa Inês (IFxSI), males and females of four slaughter weight groups of 15, 25, 35 and 45kg. The breed group presented differences of $\mathrm{pH}$ in the $\mathrm{LD}$ and $\mathrm{SM}$ muscles $(\mathrm{P}<0,05)$. The breed group BgxSI showed higher $\mathrm{pH}$ than breed group IFxSI. The color, the gender factor presented no significant effect differences for color, however breed group had effect: $\mathrm{L}^{*}(\mathrm{P}<0.001)$ of 35.25 and 32.89 for breed group IFxSI and BgxSI, respectively; and value $\mathrm{a}^{*}(\mathrm{P}<0.001)$ of 16.09 and 14.64 for breed group BgxSI and IFxSI, respectively. In the cooking losses LD and SM muscles showed no significant effect of the breed group, sex and slaughter weight group. The factors studied shear force had no effect on SM muscle. However, the slaughter weight group had effect on the shear force $(P<0.005)$ of the LD muscle, with slaughter weight groups of 15 and $25 \mathrm{~kg}$ showing higher shear force (13.57 and 10.98kgf, respectively) than slaughter weight groups of 35 and $45 \mathrm{~kg}$ (8.56 and $7.97 \mathrm{kgf}$, respectively).

Keywords: sheep; meat quality; shear force.

\section{1 - INTRODUÇÃO}

Os atributos de qualidade de carne apresentam grandes variações. Essas alterações influenciam a preferência dos consumidores e, dentre os atributos que se relacionam com a aceitação da carne, a cor é associada com o frescor do corte e a idade de abate do animal, a maciez determina a aceitação do corte, e, a perda de peso por cozimento é associada ao rendimento após o preparo.

As características de cor, maciez e rendimento são influenciados por fatores: ante mortem (sexo, raça, idade de abate e alimentação) e post mortem (tempo e temperatura de resfriamento e armazenamento) $[1,4,5$, $12,14,16,17,27,35]$. Considerando os aspectos ante mortem, DRANSFIELD, NUDE \& HOGG [10] descreveram que os efeitos da raça apresentam baixa intensidade e podem ser explicados por diferenças na maturidade em decorrência de maior ou menor precocidade e

\footnotetext{
Recebido para publicação em 28/11/2002. Aceito para publicação em 03/11/2004 (001012).

2. Departamento de Ciências dos Alimentos, Uiversidade Federal de Lavras - UFLA. Caixa Postal 37, CEP: 37200-000, Lavras-MG 3. Departamento de Zootecnia, Uiversidade Federal de Lavras - UFLA. * A quem a correspondência deve ser enviada.
}

que o peso ao abate pode afetar a qualidade, desde que a diferença entre os pesos seja suficiente para influenciar na maturidade fisiológica do animal. Entretanto, SAÑUDO et al. [25] citam que carcaça que varia de 8-27kg apresentam, nessa faixa, variações sobre a cor, a maciez, a perda de peso por cocção e o sabor.

A influência de fatores como grupos genéticos, sexo e grupo de peso ao abate sobre a qualidade de carne de ovinos foi estudada em algumas raças por: SINNETTSMITH et al. [32] (East Friesland, Oxford e Texel); DRANSFIELD, NUTE \& HOGG [10] (Dorset e Suffolk); HORCADA [12] (aragonesa Espanhola e Lacha); e, por BRESSAN et al. [8] que avaliaram carnes de cordeiros de 15, 25, 35 e 45kg das raças Bergamácia e Santa Inês). Entretanto, não existem trabalhos que avaliam o efeito da utilização da Santa Inês em cruzamentos com raças que apresentam maior desenvolvimento corporal sobre a qualidade da carne.

A raça Santa Inês tem mostrado, nos últimos anos, uma ampla expansão nas regiões Norte e Nordeste do Brasil, pois, além da aptidão para a produção de carne e pele, essa raça demonstra boa adaptação a regiões áridas [3].

O objetivo deste estudo foi avaliar o efeito dos fatores grupo genético, sexo e grupo de peso ao abate sobre o pH post mortem, a cor, a perda de peso por cozi- 
mento e força de cisalhamento nos músculos semimembranosus e longissimus dorsi.

\section{2 - MATERIAL E MÉTODOS}

\section{1 - Animais e tratamentos}

Foram utilizados 49 cordeiros machos inteiros e fêmeas (22 e 27, respectivamente), provenientes dos grupos genéticos Ile de France x Santa Inês (IFxSI) e Bergamácia x Santa Inês (BgxSI). A distribuição dos cordeiros de cada grupo genético e cada sexo foi feita aleatoriamente para a composição dos diferentes grupos de pesos ao abate (15; 25; 35 e 45kg).

Os animais entraram no experimento aos $15 \mathrm{~kg}$ e permaneceram em confinamento até alcançar 25, 35 e $45 \mathrm{~kg}$ (sendo que um lote foi abatido com $15 \mathrm{~kg}$ no início do período de confinamento). Os cordeiros receberam a ração com os componentes em proporções recomendadas pelo NATIONAL RESEARCH COUNCIL [18], com a evolução de peso controlada.

Os cordeiros, no pré-abate, submetidos a jejum de 16 horas, foram abatidos após o atordoamento mecânico, por corte transversal na região do pescoço. Em seguida, foram procedidas a esfola, a evisceração e o resfriamento da carcaça em câmara fria a $2^{\circ} \mathrm{C}$ por 24 horas.

\section{2 - Coleta de amostras}

As amostras foram retiradas das meias carcaças esquerdas após a desossa e congeladas a $-10^{\circ} \mathrm{C}$. As amostras utilizadas nas análises foram descongeladas a $4^{\circ} \mathrm{C}$ por 24 horas.

\section{3 - Análises laboratoriais}

As análises de cor, perda de peso por cozimento (PPC) e força de cisalhamento ( $\mathrm{FC}$ ) foram realizadas no Laboratório de Certificação da Qualidade de Carne e Derivados do Centro de Tecnologia de Carnes do Instituto de Tecnologia de Alimento (Campinas - SP).

\subsection{1 - Evolução do pH post mortem (pm)}

Nessa análise foi usado o potenciômetro digital (Digimed DM-20) com sensibilidade de 0,01 unidades de $\mathrm{pH}$, dotado de eletrodo de vidro e dispositivo calibrador de temperatura. As leituras foram realizadas nos músculos longissimus dorsi (LD) no espaço entre a $11^{\text {a }}$ e $12^{\text {a }}$ costela e no semimembranosus (SM) em sua porção posterior. As leituras de $\mathrm{pH}$ foram tomadas as 2, 6, 12 e $24 \mathrm{~h}$ pm, de forma que, os dados obtidos nesses horários foram submetidos à análise estatística. Os valores de $\mathrm{pH}$ final (24h pm), também foram submetidos à análise estatística separadamente.

\subsection{2 - Coloração}

A cor foi determinada pelo Sistema CIELAB $\left(\mathrm{L}^{*}=\right.$ luminosidade, $\mathrm{a}^{*}=$ teor de vermelho e $\mathrm{b}^{*}=$ teor de amarelo) com colorímetro (Minolta CR-200b). As amostras dos músculos LD e SM foram divididas em fatias de $2,5 \mathrm{~cm}$ e após $30 \mathrm{~min}$ foram executadas as leituras em 3 pontos distintos em cada fatia. A média de cada componente de cor, por fatia, foi usada na análise estatística.

\subsection{3 - Perda de peso por cozimento (PPC)}

$\mathrm{Na}$ análise da PPC foram empregadas as amostras de músculos LD e SM utilizadas na análise de cor. As amostras foram pesadas e envolvidas em papel alumínio e colocadas para assar em chapa pré-aquecida a $150^{\circ} \mathrm{C}$. Ao alcançar a temperatura interna de $35^{\circ} \mathrm{C}$, as amostras foram viradas e mantidas até atingir a temperatura interna de $72^{\circ} \mathrm{C}$. Em seguida, as mesmas foram resfriadas a temperaturas ambientes. A PPC para cada músculo foi calculada pela média (\%) das diferenças de pesos entre as amostras antes do cozimento e após o resfriamento [2].

\subsection{4 - Força de cisalhamento (FC)}

Para determinar a FC foram utilizadas as amostras da PPC. De cada fatia foram extraídos, no sentido das fibras, 4 cilindros com $12,7 \mathrm{~mm}$ de diâmetro e $20 \mathrm{~mm}$ de comprimento, utilizando uma sonda acoplada a uma furadeira. A FC foi medida pela secção dos cilindros, usando o Instron M1122, acoplado ao Warner-Blatzer, com a escala variando de 0 a 50kgf [7].

\subsection{5 - Delineamento experimental e análise es- tatística}

O experimento foi organizado em delineamento inteiramente casualizado, em fatorial com 16 tratamentos, sendo 2 grupos genéticos (IFxSI e BgxSI), 2 sexos (machos inteiros e fêmeas) e 4 grupos de peso ao abate $(15,25,35$ e $45 \mathrm{~kg})$. Cada tratamento foi composto de 2 a 5 parcelas e cada parcela de 1 animal. Os resultados das diferentes variáveis foram submetidos à análise de variância com o programa SAS [28]. Os resultados significativos para os grupos de peso foram submetidos a teste de média [29].

\section{3 - RESULTADOS E DISCUSSÃO}

\section{1 - Evolução do pH post mortem (pm) nos múscu- los SM e LD}

As médias de $\mathrm{pH}$, obtidas as 2, 6, 12 e 24 horas pm, revelaram diferenças $(\mathrm{P}<0,01)$ entre os horários e quando os dados foram submetidos à análise de regressão mostraram um curva ajustada com um coeficiente de determinação $\left(\mathrm{R}^{2}\right)$ de 0,9843 (Figura 1). Essa curva mostra que houve uma maior velocidade de declínio do $\mathrm{pH}$ nos três primeiros horários e tendência à estabilização a partir de 12 horas. Essa queda de $\mathrm{pH}$ é resultado da utilização das reservas de glicogênio via glicólise pm que tem como produto final o ácido lático. A velocidade de queda do $\mathrm{pH}$ e o $\mathrm{pH}$ final podem afetar as estruturas protéicas da carne, alterando a coloração, o brilho superficial, a capacidade de retenção de água, o rendimento no cozimento e a maciez [11, 35]. 


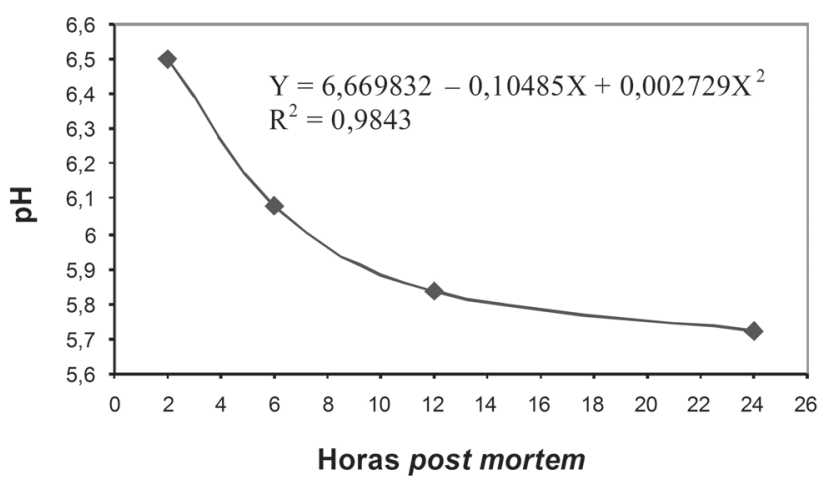

FIGURA 1. Curva de declínio do pH nos músculos longissimus dorsi e semimembranosus.

Os fatores grupos genéticos, pesos ao abate e músculos influenciaram $(\mathrm{P}<0,01)$ as médias de $\mathrm{pH}$ obtidos no post mortem (médias dos horários 2, 6, 12 e 24 horas) e sobre as médias de $\mathrm{pH}$ final. Entretanto, o fator sexo não afetou as médias de $\mathrm{pH}$ e o $\mathrm{pH}$ final (Tabela 1). Interação significativa $(\mathrm{P}<0,01)$ foi encontrada entre os fatores grupos genéticos e pesos ao abate (Tabela 2).

TABELA 1. Médias de pH nos músculos semimembranosus e longissimus dorsi.

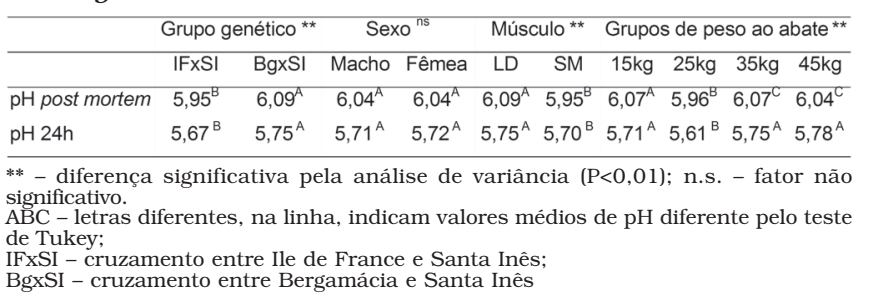

Considerando os grupos genéticos (IFxSI e BgxSI com $\mathrm{pH}$ de 5,95 e 6,09 e pH 24 h de 5,67 e 5,75, respectivamente), os cordeiros IFxSI mostraram reações bioquímicas de acidificação da carne mais rápidas do que aqueles BgxSI, bem como uma maior acidificação da carne às $24 \mathrm{~h}$ post mortem. Essas informações sugerem que, no momento do abate, os ovinos IFxSI possivelmente apresentaram reservas de glicogênio muscular mais elevadas, do que os ovinos BgxSI. Vários autores estudando o efeito de raças sobre o comportamento de $\mathrm{pH}$ post mortem não relataram, em ovinos, diferenças significativas: SINNETT-SMITH et al. [32], utilizando as raças East Friesland, Oxford e Texel; e DRANSFIELD, NUTE \& HOGG [10], as raças Dorset e SuffolK. Entretanto, confirmando os resultados encontrados no presente experimento: BRESSAN et al. [8] relataram em ovinos das raças Santa Inês e Bergamácia, curvas de pH que diferiram, de forma que a instalação do rigor mortis foi mais rápida em músculos de animais da raça Bergamácia; e, HORCADA et al. [12] encontraram médias de pH final elevado na raça Aragonesa Espanhola, quando comparada à raça Lacha, em cordeiros de $24 \mathrm{~kg}$. Essa diferença para $\mathrm{pH}$ post mortem entre raças ou cruzamento é atribuído, normalmente, a variações entre grupos genéticos a susceptibilidade ao estresse préabate [1 1, 35].
A diferença para média de $\mathrm{pH}$ e $\mathrm{pH}$ final nos músculos LD e SM $(6,09$ e 5,95; 5,75 e 5,70, respectivamente) demonstrou que a instalação do rigor mortis foi mais rápida no músculo $\mathrm{SM}$ do que no $\mathrm{LD}$; e a acidificação do músculo da perna foi mais intensa do que no músculo do lombo. Os conhecimentos clássicos de morfologia descrevem que os músculos apresentam distintas composições em quantidades de fibras vermelhas, intermediárias e brancas em decorrência das funções dos grupos musculares em vida [35]. Assim, embora a carne de ovinos seja considerada como carne vermelha, os grupos musculares que compõem o lombo apresentam uma maior quantidade de fibras brancas (metabolismo glicolítico), do que os grupos musculares da perna (metabolismo oxidativo) [11, 35]. Os músculos com maiores quantidades de fibras brancas, segundo LAWRIE [17], apresentam uma maior concentração de glicogênio, do que aqueles com maiores quantidades de fibras brancas, causando no post mortem uma maior produção de ácido lático, e maior acidificação da carne. Entretanto, esse raciocínio não descreve os resultados encontrados no presente trabalho. Nesse caso, é possivel que a maior acidificação no músculo da perna, do que no lombo seja decorrência das diferenças morfológicas e vias de utilização das reservas energéticas, de forma que: no ante mortem, o músculo LD utilizou preferencialmente o metabolismo glicolítico e suas reservas de glicogênio e o músculo SM usou preferencialmente o metabolismo oxidativo; e, no post mortem, em que a energia é fornecida pela via glicolítica (ausência de oxigênio), os músculos da perna tenham apresentado uma reserva energética, na forma de glicogênio.

$\mathrm{O}$ teste de média identificou diferença no $\mathrm{pH}$ médio entre os grupos de peso ao abate (Tabela 1), em que o grupo de $25 \mathrm{~kg}$ mostrou $\mathrm{pH}$ mais baixo $(5,96)$ do que os grupos de 15, 35 e 45kg (6,07; 6,07 e 6,04, respectivamente). Comportamento semelhante foi verificado para $\mathrm{pH}$ final entre os grupos de peso avaliados. Esses resultados discordam daqueles observados por BRESSAN et al. [8], que comparando cordeiros de 15, 25, 35 e $45 \mathrm{~kg}$ das raças Bergamácia e Santa Inês, observaram declínio de $\mathrm{pH}$ mais rápido em animais de 35 e 45kg, cujo resultado foi atribuído a presença de gordura subcutânea que possivelmente tenha atuado como isolante térmico, gerando temperaturas post mortem no interior das massas musculares mais elevadas, que acelerou a glicólise [13]. Entretanto, no presente experimento, o comportamento de $\mathrm{pH}$ do grupo de $25 \mathrm{~kg}$ pode ser justificado pelo fator genético, pois foram utilizados ovinos oriundos de cruzamentos de raças com diferentes aptidões [3] que apresentam diferentes curvas de crescimento do tecido muscular e gordura [22] e com diferentes padrões de deposição de gordura na carcaça [20].

A interação entre peso ao abate e grupos genéticos (Tabela 2) mostrou que, nos animais de 15 e $25 \mathrm{~kg}$, as médias de $\mathrm{pH}$ entre animais IFxSI e BgxSI diferem significativamente, sendo que os animais IFxSI mostram $\mathrm{pH}$ final mais baixo do que os BgxSI. Esse comportamento também é verificado nos animais de $45 \mathrm{~kg}$, embora sem diferença significativa. 
TABELA 2. Médias de pH final para a interação entre grupos genéticos e pesos ao abate.

\begin{tabular}{ccccc}
\hline & \multicolumn{4}{c}{ Peso ao abate } \\
\hline Grupos genético & $15 \mathrm{~kg}$ & $25 \mathrm{~kg}$ & $35 \mathrm{~kg}$ & $45 \mathrm{~kg}$ \\
\hline IFxSI & $5,63^{\mathrm{B}}$ & $5,56^{\mathrm{C}}$ & $5,77^{\mathrm{A}}$ & $5,73^{\mathrm{A}}$ \\
BgxSI & $5,80^{\mathrm{A}}$ & $5,67^{\mathrm{B}}$ & $5,73^{\mathrm{A}}$ & $5,83^{\mathrm{A}}$
\end{tabular}

$\mathrm{AB}$ - letras diferentes na linha indicam valores médios de $\mathrm{pH}$ diferente pelo teste $\mathrm{Scott}$ e Knott;

IFxSI - cruzamento entre Ile de Francis e Santa Inês;

BgxSI - cruzamento entre Bergamácia e Santa Inês:

As médias de pH final, neste trabalho, oscilaram entre 5,56 a 5,83. Em cordeiros são citadas variações entre 5,5 a $5,9[21,23,31]$ e são considerados valores normais para carne vermelha, pH entre 5,5 a 5,8 [11].

\section{2 - Cor nos músculos SM e LD (L*, a*, b*)}

\subsection{1 - Índice $L^{*}$ (luminosidade) nos músculos SM e LD}

A análise de variância indicou que o grupo genético e peso ao abate influenciaram $(\mathrm{P}<0,001)$ a luminosidade. Entretanto, o fator sexo não influenciou o L* (Tabela 3).

Com relação aos grupos genéticos, observa-se que ovinos IFxSI mostraram L* mais elevado do que os ovinos BgxSI com médias de 35,25 e 32,89, respectivamente no músculo $\mathrm{SM}$, e de 35,02 e 33,06, respectivamente, no músculo LD. Esses resultados confirmam os achados de PRADO [23] que, comparando L* de cordeiros Santa Inês com cordeiros Bergamácia, verificou maior luminosidade nos animais Bergamácia; SAÑUDO et al. [26] encontraram $L^{*}$ mais baixo em ovinos da raça Castelana, do que em cordeiros da raça Manchega e Awassi. Esses autores compararam, nos respectivos trabalhos, animais de mesma idade e submetidos a condições zootécnicas e manejos de abate semelhantes. Entretanto, como as raças comparadas apresentam aptidões distintas, é possível que o tecido muscular apresente adaptações em função de demandas energéticas, determinando significativas diferenças com relação à luminosidade de amostras.

TABELA 3. Médias para os componentes de cor ( $\left.\mathrm{L}^{*}, \mathrm{a}^{*}, \mathrm{~b}^{*}\right)$ nos músculos SM e LD de cordeiros.

\begin{tabular}{|c|c|c|c|c|c|c|}
\hline Músculos & Fatores & Tratamento & $\mathrm{n}$ & $\mathrm{L}^{*}$ & $a^{*}$ & $b^{*}$ \\
\hline \multirow{8}{*}{ SM } & \multirow{2}{*}{ Grupo Genético } & $\mathrm{IFxSI}$ & 16 & $35,25^{a}$ & $14,64^{\circ}$ & $4,82^{a}$ \\
\hline & & BgxSI & 28 & $32,89^{b}$ & $16,09^{a}$ & $3,86^{b}$ \\
\hline & \multirow{2}{*}{ Sexo } & Macho & 20 & $34,13^{a}$ & $15,32^{a}$ & $4,32^{a}$ \\
\hline & & Fêmea & 24 & $33,43^{a}$ & $15,77^{\mathrm{a}}$ & $4,11^{\mathrm{a}}$ \\
\hline & \multirow{4}{*}{ Peso ao abate } & $15 \mathrm{~kg}$ & 11 & $38,00^{a}$ & $12,27^{d}$ & $4,76^{\mathrm{a}}$ \\
\hline & & $25 \mathrm{~kg}$ & 11 & $33,41^{\mathrm{b}}$ & $15,17^{c}$ & $3,89^{\mathrm{a}}$ \\
\hline & & $35 \mathrm{~kg}$ & 11 & $32,22^{c}$ & $16,81^{\mathrm{b}}$ & $4,07^{\mathrm{a}}$ \\
\hline & & $45 \mathrm{~kg}$ & 11 & $31,36^{\mathrm{C}}$ & $18,01^{a}$ & $4,13^{\mathrm{a}}$ \\
\hline \multirow{8}{*}{ LD } & \multirow{2}{*}{ Grupo Genético } & IFxSI & 16 & $35,02^{a}$ & $13,95^{\circ}$ & $4,40^{a}$ \\
\hline & & BgxSI & 28 & $33,06^{b}$ & $15,03^{\mathrm{a}}$ & $4,24^{\mathrm{a}}$ \\
\hline & \multirow{2}{*}{ Sexo } & Macho & 20 & $34,19^{a}$ & $14,27^{a}$ & $4,07^{a}$ \\
\hline & & Fêmea & 24 & $33,45^{a}$ & $14,93^{\mathrm{a}}$ & $4,49^{\mathrm{a}}$ \\
\hline & \multirow{4}{*}{ Peso ao abate } & $15 \mathrm{~kg}$ & 11 & $38,25^{a}$ & $10,83^{\circ}$ & $5,65^{a}$ \\
\hline & & $25 \mathrm{~kg}$ & 11 & $33,64^{b}$ & $14,84^{b}$ & $4,02^{b}$ \\
\hline & & $35 \mathrm{~kg}$ & 11 & $32,65^{\mathrm{b}}$ & $15,80^{b}$ & $4,22^{b}$ \\
\hline & & $45 \mathrm{~kg}$ & 11 & $30,58^{c}$ & $17,05^{a}$ & $3,34^{b}$ \\
\hline
\end{tabular}

ab - Letras diferentes na coluna, significa diferença entre as médias a nível de $(\mathrm{P}<0,05)$

$\mathrm{L}^{*}=$ luminosidade; $\mathrm{a}^{*}=$ tendência para o vermelho; $\mathrm{b}^{*}=$ tendência para o amarelo. $\mathrm{n}=$ número de unidades experimentais.
Considerando o fator peso ao abate, observa-se, nos músculos SM e LD, que os animais de $15 \mathrm{~kg}$ apresentaram L* mais elevado, enquanto os animais de $45 \mathrm{~kg}$ mostraram $L^{*}$ mais baixo. PRADO [23] e PEREZ et al. [21], trabalhando com cordeiros 15, 25, 35 e 45kg, verificaram maior $\mathrm{L}^{*}$ em cordeiros de $15 \mathrm{~kg}$. Esses autores descrevem que, com o aumento de peso, ocorre variação na composição química da carne (redução na umidade e aumento na gordura), assim, animais mais jovens com maior \% de água no tecido muscular, resultaram $\mathrm{L}^{*}$ mais elevado.

As médias de luminosidade ( $\mathrm{L}^{*}$ ) variaram de 31,36 a 38,00 no músculo SM e de 30,58 a 38,25 no músculo LD. Em ovinos, são citadas variações de 30,03 a 49,47 $[10,23,25,26,33,34]$.

\subsection{2 - Índice $\mathrm{a}^{*}$ (tendência para o vermelho)}

A análise de variância indicou que os grupos genéticos GP $(\mathrm{P}<0,001)$ e grupos de peso ao abate $(\mathrm{P}<0,001$ para $\mathrm{SM}$ e $\mathrm{P}<0,05$ para $\mathrm{LD}$ ) afetaram o teor de vermelho. Enquanto que o sexo não influenciou o valor $a^{*}$ (Tabela 3).

Analisando o efeito do grupo genético, os cordeiros BgxSI apresentam maior teor de vermelho do que os animais IFxSI. Nesses grupos foi observado também que ovinos BgxSI apresentaram pH mais elevado $(5,67)$ do que os IFxSI $(5,75)$. HEDRICK et al. [11] descreveram que carnes com $\mathrm{pH}$ mais elevado às 24 horas post mortem resulta em carnes mais escuras. Entretanto, SAINZ [24] menciona que a variação no teor de vermelho ( $\mathrm{a}^{*}$ ) pode estar relacionada com a capacidade do grupo genético em influenciar a proporção entre as formas de mioglobina (desoximioglobina e oximioglobina). Contrariando esses resultados, PRADO [23] e PEREZ et al. [21] trabalhando com os grupos genéticos SI e Bg e, SAÑUDO et al. [26] avaliando os cordeiros Churra, Castelana, Manchega e Awassi, não verificaram diferenças no teor de vermelho entre raças ou grupos genéticos.

As médias de $\mathrm{a}^{*}$ foram mais elevadas à medida que os animais aumentaram de peso. Esse comportamento foi verificado no músculo SM $(12,27 ; 15,17$; 16,81 e 18,01 para animais de $15,25,35$ e $45 \mathrm{~kg}$, respectivamente) e no músculo LD $(10,83 ; 14,84 ; 15,80$ e 17,05 para animais de $15,25,35$ e $45 \mathrm{~kg}$, respectivamente). Resultado semelhante foi observado por PRADO [23] trabalhando com animais puros SI e Bg. Esse autor justifica o aumento no teor de vermelho em função do aumento de peso em decorrência do aumento na concentração de pigmentos hêmicos à medida que aumenta o peso ao abate $[4,5]$. Em trabalhos que compararam cordeiros de 21,7 e $27,8 \mathrm{~kg}$ e 10 e $12 \mathrm{~kg}$ não foi observada diferença para o teor de vermelho [33; 34].

As médias de $\mathrm{a}^{*}$ variaram de 12,27 a 18,00 para o músculo SM e de 10,83 a 17,05 em LD. Variações médias entre 8,24 a 23,53 foram relatadas para ovinos [10, $23,26,33,34]$. 


\subsection{3 - Índice $b^{*}$ (tendência para 0 amarelo)}

Os grupos genéticos afetaram o teor de amarelo $(\mathrm{P}<0,05)$ no músculo SM. Cordeiros IFxSI apresentaram tendência para o amarelo mais elevada $(4,82)$, do que os cordeiros SIxBg $(3,86)$. Entretanto, esse comportamento não aparece quando comparado o valor $\mathrm{b}^{*}$ no músculo LD.

Comparando ovinos de diferentes raças, SAÑUDO et al. [25] encontraram índices de $b^{*}$ mais elevados nos cordeiros Churra e Manchega, do que em cordeiros Manchega, Castelana e Awassi. Por outro lado, PRADO [23] encontrou resultados semelhantes em cordeiros puros das raças Santa Inês e Bergamácia.

$\mathrm{O}$ valor $\mathrm{b}^{*}$ normalmente determina o teor de amarelo, que é influenciado pela presença de betacaroteno na gordura [26]. Como no presente estudo, o percentual de extrato etéreo (\%) foi semelhante e os animais receberam a mesma alimentação, é possivel que essa diferença seja resultado de uma maior aptidão na deposição de betacaroteno [10] entre os animais dos grupos genéticos em questão.

As médias de b* variaram entre 3,34 e 5,65. Em carne ovina são citadas médias de 3,38 a 11,10 [10, 23, 26, $34,35]$.

\section{3 - Força de cisalhamento (FC) nos músculos SM e LD}

A força de cisalhamento não foi influenciada pelos grupos genéticos, sexo e pesos ao abate (músculo SM). Entretanto, os pesos ao abate afetaram $(\mathrm{P}<0,05)$ a $\mathrm{FC}$ no músculo LD (Tabela 4). Houve interação para FC no músculo SM $(\mathrm{P}<0,05)$ entre os fatores sexo e peso ao abate. E houve interação entre grupos genéticos e peso ao abate $(\mathrm{P}<0,05)$ no músculo LD.

Analisando os fatores sexo e peso ao abate sobre a $\mathrm{FC}$, observa-se que em machos e fêmeas, os animais de $15 \mathrm{~kg}$ apresentaram uma FC mais elevada, do que os animais de 25, 35 e 45kg. Porém, esse comportamento não foi observado em cordeiros fêmeas, quando analisado o músculo SM.

Considerando os fatores grupo genético e peso ao abate sobre a FC, os cordeiros BgxSI de 15 e 25kg mostraram, no músculo LD, médias mais elevadas $(15,75$ e 12,48kgf, respectivamente), do que os animais de 35 e $45 \mathrm{~kg}(7,30$ e $8,08 \mathrm{kgf}$, respectivamente). Esse comportamento da FC foi observado no mesmo grupo genético no músculo SM (embora sem diferença significativa) e nos animais IFxSI as médias de FC foram semelhantes.

Avaliando os fatores isolados, a ausência de efeito de raças ou grupo genético sobre a maciez em ovinos foi descrita: por SAÑUDO et al. [26] que utilizaram as raças Churra, Castelana, Manchega e Awassi; por BRESSAN et al. [8] que estudaram animais puros das raças Santa Inês e Bergamácia; por NOTTER, KELLY \& BERRY [19] que utilizaram animais cruzados $1 / 2$ Suffolk, $1 / 2$ Rambouillet; $1 / 2$ Suffolk, $1 / 4$ Finnsheep, $1 / 4$ Rambouillet ou
Dorset; e por KISHORE, RAWAT \& BASUTHAKUR [15] que compararam animais Dorset x Avikalin e Avikalin. A ausência de efeito do sexo sobre a FC foi relatada por VELASCO et al. [33]; VERGARA, MOLINA \& GALLEGO [34]; e por KOOHMARAIE, SHAKELFORD \& WHEELER [16].

TABELA 4. Médias de força de cisalhamento (kgf) em músculos semimembranosus (SM) e longissimus dorsi (LD).

\begin{tabular}{|c|c|c|c|c|c|c|c|c|}
\hline \multirow{3}{*}{$\begin{array}{l}\text { Grupo } \\
\text { de peso }\end{array}$} & \multicolumn{4}{|c|}{ Sexos } & \multicolumn{4}{|c|}{ Grupos genéticos } \\
\hline & \multicolumn{2}{|c|}{ Machos } & \multicolumn{2}{|c|}{ Fêmeas } & \multicolumn{2}{|c|}{ IFxSI } & \multicolumn{2}{|c|}{$\mathrm{BgxSI}$} \\
\hline & SM & LD & SM & LD & SM & LD & SM & LD \\
\hline $15 \mathrm{~kg}$ & $9,12^{a}$ & $12,4^{a}$ & $7,64^{D}$ & $13,20^{a}$ & $8,12^{a}$ & $9,85^{a}$ & $8,64^{a}$ & $15,74^{a}$ \\
\hline $25 \mathrm{~kg}$ & $7,25^{b}$ & $9,15^{a}$ & $11,76^{a}$ & $12,27^{\mathrm{a}}$ & $9,50^{\mathrm{a}}$ & $8,93^{a}$ & $9,51^{a}$ & $12,48^{a}$ \\
\hline $35 \mathrm{~kg}$ & $7,56^{\mathrm{b}}$ & $9,05^{\mathrm{a}}$ & $7,33^{b}$ & $8,41^{a}$ & $8,00^{a}$ & $10,16^{a}$ & $6,90^{\mathrm{a}}$ & $7,30^{b}$ \\
\hline $45 \mathrm{~kg}$ & $7,54^{b}$ & $10,94^{\mathrm{a}}$ & $8,01^{b}$ & $5,92^{a}$ & $8,16^{\mathrm{a}}$ & $8,77^{\mathrm{a}}$ & $7,39^{\mathrm{a}}$ & $8,08^{b}$ \\
\hline
\end{tabular}

${ }^{a b}=$ letras diferentes representam diferenças significativas dentro das colunas $(\mathrm{P}<0,05)$.

Em estudos de efeito de peso ao abate ou idade ao abate, os resultados são contraditórios. BERGE et al. [4, 5], trabalhando com ovinos de: 1,$7 ; 2,8 ; 4,3 ; 7$ e 12 meses, descrevem que animais mais velhos ou pesados mostram maior FC que animais mais jovens. Por outro lado, alguns autores não encontraram diferença em ovinos de diferentes pesos ao abate, tais como: VELASCO et al. [33] no músculo LD de cordeiros da raça Talaverana com 10 e 12kg; e BRESSAN et al. [8] no músculo SM com animais de peso ao abate de 15, 25, 35 e $45 \mathrm{~kg}$. Entretanto, SAÑUDO et al. [25], analisando a maciez de 3 diferentes GP de cordeiros $(8,07 ; 10,22$ e $13,42 \mathrm{~kg}$ ), encontraram FC mais elevada para cordeiros de peso intermediário e valores iguais para os pesos extremos e os autores justificaram que o aumento da maciez também está relacionado com os níveis de gordura da carcaça [31].

Estudando a maciez de carnes de cordeiros vendidas em supermercados da Nova Zelândia, BICKERSTAFFE, LE COUTEUR \& MORTON [6], verificaram que amostras com FC acima de $11 \mathrm{kgf}$ apresentaram níveis de aceitação reduzida e definiram essas carnes como "duras". No presente trabalho, foram encontradas médias de FC de: 11,$76 ; 12,27 ; 12,48 ; 13,20$ e 15,74kgf para animais de 15 e $25 \mathrm{~kg}$, respectivamente. Ou seja, carnes de baixa maciez, seguindo o limite de $11 \mathrm{kgf}$, ocorreram em animais de menor peso sem gordura de cobertura. Assim, esses valores elevados de FC podem ser conseqüência do encurtamento de sarcômeros, pois as carcaças foram resfriadas a partir de $2 \mathrm{~h}$ pm em temperaturas de $2^{\circ} \mathrm{C}$ (por 24h), determinando a condição conhecida como "cold shortening" nas carcaças de cordeiros de baixos pesos (sem gordura de cobertura) e, mais susceptiveis ao encurtamento e endurecimento da carne após o cozimento [9, 11].

As médias para FC variaram de 7,25 a 11,76 e de 5,92 a 15,74kgf para os músculos SM e LD, respectivamente. Na literatura grandes variações são encontradas nos resultados entre os autores, desde médias de 2,5 a $15,10 \mathrm{kgf}[16,23,26]$. 


\section{4 - Perda de peso por cozimento (PPC)}

As análises de variância dos dados de PPC não identificaram diferenças entre os grupos genéticos, sexo e grupos de peso ao abate, indicando que as médias foram semelhantes quando comparados esses fatores (Tabela 5).

Os grupos genéticos não manifestaram efeito significativo sobre a PPC. Resultados semelhantes foram relatados em ovinos por: BRESSAN et al. [8] e DRANSFIELD, NUTE \& HOGG [10]. Com relação ao sexo, vários autores não encontraram efeito sobre a PPC, tais como: VERGARA et al. [34] em cordeiros da raça Manchego; VELASCO et. al. [33] em ovinos da raça Talaverana; e, DRANSFIELD, NUTE \& HOGG [10] em cordeiros Dorset dawn e Suffolk.

TABELA 5. Médias para perda de peso por cozimento (\%) de ovinos em crescimento.

\begin{tabular}{cccccc}
\hline Fatores & Grupo & $\mathrm{n}$ & semimembranosus. & $\mathrm{n}$ & longissimus dorsi \\
\hline \multirow{2}{*}{ Grupos genéticos } & IFxSI & 17 & $34,94^{\mathrm{a}}$ & 18 & $36,70^{\mathrm{a}}$ \\
& BgxSI & 29 & $35,40^{\mathrm{a}}$ & 31 & $37,38^{\mathrm{a}}$ \\
\hdashline Sexos & Macho & 22 & $35,63^{\mathrm{a}}$ & 22 & $37,12^{\mathrm{a}}$ \\
& Fêmea & 24 & $34,86^{\mathrm{a}}$ & 27 & $37,13^{\mathrm{a}}$ \\
\hdashline Peso & $15 \mathrm{~kg}$ & 12 & $35,94^{\mathrm{a}}$ & 12 & $38,70^{\mathrm{a}^{\mathrm{a}}}$ \\
ao & $25 \mathrm{~kg}$ & 12 & $34,01^{\mathrm{a}}$ & 13 & $37,40^{\mathrm{a}}$ \\
abate & $35 \mathrm{~kg}$ & 11 & $35,55^{\mathrm{a}}$ & 12 & $37,12^{\mathrm{a}}$ \\
& $45 \mathrm{~kg}$ & 11 & $35,47^{\mathrm{a}}$ & 12 & $35,39^{\mathrm{a}}$ \\
\hline
\end{tabular}

${ }^{\mathrm{a}}=$ Todas as médias foram semelhantes $(\mathrm{P}<0,05)$

$\mathrm{n}=$ número de unidades experimentais.

Considerando o fator peso ao abate, a PPC não foi influenciada. Comportamento semelhante foram descritos por BRESSAN et al. [8] e VELASCO et al. [33]. Por outro lado, SHACKELFORD, WHEELER \& KOOHMARAIE [30] e KEMP et al. [14] relatam aumento na PPC com o aumento do peso ao abate e justificam que esse aumento na PPC é resultado do aumento no teor de gordura na carne que, quando aquecida para a quantificação da perda por cocção, parte da gordura se perde constituindo parte da PPC.

As médias de PPC variaram de 33,31 a 37,93\%. Valores mais elevados (36,30 e 38,0\%) foram descritos por SHACKELFORD, WHEELER \& KOOHMARAIE [30] e mais baixos $(28,12$ e $29,15 \%)$ por VELASCO et al. [33].

\section{4 - CONCLUSÕES}

O pH final em ovinos é variável em função do grupo genético, músculo estudado e peso ao abate.

Os ovinos do grupo genético Ile de France x Santa Inês apresentam amostras de carnes com coloração mais clara (luminosidade mais elevada e teor de vermelho mais baixo) do que ovinos Bergamácia x Santa Inês (luminosidade mais baixa e teor de vermelho mais elevado).

A maciez no músculo semimembranosus não é afetada por grupo genético, sexo e peso ao abate. No músculo longissimus dorsi, os ovinos de 15 e $25 \mathrm{~kg}$ apresentam força de cisalhamento elevada, caracterizando carne sem maciez.

\section{5 - REFERÊNCIAS BIBLIOGRÁFICAS}

[1] ALVI, A.S. The influence of sex status on meat quality characteristics in sheep. Fleischwirtsch, Frankfurt, v. 11, n. 60 , p. 2037-2042, 1980.

[2] AMASA. Guidelines for cooking sensory evaluation of meat. American Meat Science Association, National Live Stock and Meat Board, Chicago, IL.1978.

[3] BARROS, N.N.; SIMPLÍCIO, A.A. Produção intensiva de ovinos de corte: perspectivas e cruzamentos. In: SIMPÓSIO MINEIRO DE OVINOCULTURA, 2001, Lavras. Anais... Lavras: UFLA, 2001. p. 21-48.

[4] BERGE, P.; SANCHES, A.; SEBASTIAN, I.; ALFONSO, M.; SANUDO, C. Lamb meat texture as influenced by age and collagen characteristics. 44th ICoMST, n. 44, p. 304-305, 1998

[5] BERGE, P.; SANCHEZ, A.; DRANSFIELD, E.; SEBASTIAN, I.; SANUDO, C.; BAYLE, M.C. Variations of meat composition and quality in different commercial lamb types. 45th ICoMST v. 45, n. 45, p. 502-503, 1999.

[6] BICKERSTAFFE, R.; LE COUTEUR, C.E.; MORTON, J.D. Consistency of tenderness in New Zealand retail meat. 44th ICoMST, n. 43, p. 196-197, 1997.

[7] BLATZLER, L.J. Característica Organoléptica de la carne In: PRICE, J.F.; SCHWEIGERT, B.S. Ciencia de la Carne y de los productos carnicos. Cap. 3, p. 125138. Tradução de FUENTE J. L. Zaragoza: ACRIBA S. A. $2^{\text {a }}$ ed. 1994.

[8] BRESSAN, M.C.; PRADO, O.V.; PÉREZ, J.R.O.; LEMOS, A.L.S.; BONAGURIO, S. efeito do peso ao abate de cordeiros santa Inês e Bergamácia sobre as características físico-químicas da carne. Ciênc. Tecnol. Aliment., Campinas, v. 21, p. 293-303, 2001.

[9] DEVINE, C.E.; GRAAFHUIS, A.E. The basal toughness of unaged lambs. Meat science. Essex, v., n. 39, p. 285291, 1994

[10] DRANSFIELD, E.; NUTE, G.R.; HOGG, B. W. Carcass and eating quality of ram, castred ram and ewe lambs. British Society of Animal Production. Langford, v. 50, n., p. 291-299, 1990

[11] HEDRICK, H.B.; ABERLE, E.D.; FORREST, J.C.; M.D. JUDGE; MERKEL, R.A. Properties of fresh meat. In: Principles of Meat Science ( $3^{\text {rd }}$ Ed.), Kendall/Hunt Publishing Company, Dubuque, IA. 1994. p. 123-131.

[12] HORCADA, A.; BERIAIN, M.N.J.; PURROY, A.; LIZASO, G.; CHASCO, J. Effect of sex on meat quality of spanish lamb breeds (Lacha and Rasa Aragonesa). Anim. Sci. Hamilton, v. 67, p. 541-547, 1998.

[13] JOHNSON, M.H.; BIDNER, T.D.; MCMILLIN, K.W.; DUGAS, S.M.; HEMBRY, F.G. The effect of three temperature conditioning treatments and subcutaneous fat removal on lamb quality. Journal Animal Science, Champaign, n. 67, p. 2309-2315, 1989

[14] KEMP, J.D.; JOHNSON, A.E.; STEWART, D.F.; ELY, D.G.; FOX, J.D. Effect of dietary protein, slaughter weight and sex on carcass composition, organoleptics properties and cooking losses of lamb. Journal Of Animal Science, Champaign, v. 42, p. 575-583, 1976.

[15] KISHORE, K.; RAWAT, P.S.; BASUTHAKUR. Comparative performance of terminal cross of avikalin with dorset and avikalin lambs regarding growth, feedlot and carcass characteristics. Indian J. Anim. Sci., v. 58, n. 8, p. 774-778, august, 1984.

[16] KOOHMARAIE, M.; SHAKELFORD, S.D.; WHEELER, T.L. Effects of a b-adrenergic agonist (L-644,969) and 
male sex condition on muscle growth and meat quality of callipyge lambs. Journal Animal Science. n. 74, p. 70-79, 1996.

[17] LAWRIE, R. A Meat Science. Pergamon Press, Oxford. 1985. 293p.

[18] NATIONAL RESEARCH COUNCIL (NRC). Nutrient requirements of domestic animal: Nutrient requirements of sheep. Washington, 1985, p.99.

[19] NOTTER, D.R.; KELLY, R.F.; BERRY, B.W. Effects of ewe breed and management system on efficiency of lamb production: III. Meat characteristics. Journal of Animal Science, Champaign, v. 69, p. 3523-3532, 1991.

[20] OSÓRIO, M.T.; SIERRA, I.; SAÑUDO, C.; OSÓRIO, J.C. Influência da raça, sexo e peso/idade sobre o rendimento da em cordeiros. In: Reunião da Sociedade Brasileira de Zootecnia, 35; 1998, Botucatu. Anais... Botucatu; Gnosis, 1998. 1 CD-rom.

[21] PEREZ, J.R.O.; BONAGURIO, S.; BRESSAN, M.C.; PRADO, O.V. Efeitos de dejetos de suínos na qualidade de carne de ovinos. In: Reunião da Sociedade Brasileira de Zootecnia, 34, Juiz de Fora, MG, v. 1, p. 391, 1997.

[22] PILAR, R.C. Desempenho, características de carcaça, composição e alometria dos cortes em cordeiros Merino Australiano e cruza Ile de France $x$ Merino Australiano. Lavras, 2002, 237p. Tese (Doutorado). Departamento de Zootecnia. Universidade Federal de Lavras (UFLA).

[23] PRADO, O.V. Qualidade de carne de cordeiros Santa Inês e Bergamácia abatidos em diferentes pesos. Lavras, 2000, 109p. Dissertação (Mestrado). Departamento de Zootecnia. Universidade Federal de Lavras (UFLA).

[24] SAINZ, R.D. Qualidade das carcaças e da carne bovina. In: CONGRESSO BRASILEIRO DAS RAÇAS ZEBUINAS. 27 a 30 de Outubro de 1996. Reprodução e Genética Aplicada aos Zebuínos. Anais..., 1996, p.1

[25] SAÑUdO, C.; ALFONSO, M.; SANCHES, A.; DELFA, R.; TEIXEIRA, A. Carcass and meat quality in light lambs from different fat classes in the EU carcass classification system. Meat Science. Essex, v. 56 n., p. 89-54, 2000.
[26] SAÑUDO, C.; CAMPO, M.M.; SIERRA, I.; MARIA, G.A.; OLLETA, J.L.; SANTOLARIA, P. Breed effect on carcass and meat quality of suckling lambs. Meat Science. Essex, v. 46, n. 4, p. 357-365, 1997.

[27] SAÑUDO, C.; SIERRA, I.; LOPEZ, M.; FORCADA, F. La qualité de la viande ovine. Etude des différents facteurs qui la conditionnent, EUR 11479, Commission des CE Rapport, pp. 67-81, 1986.

[28] SAS Institute Inc. SAS/ETF User's Guide, Version 6. Second Edition. Carry. NC: SAS Institute Inc., 1993.

[29] SCOTT, A.J.; KNOTT, M.A. Cluster Analysis Method for Grouping Means In the Analysis of Variance. Biometrics, Washington v. 30, p. 507-512 sept. 1994.

[30] SHACKELFORD, S.D.; WHEELER, T.L.; KOOHMARAIE, M. Effect of the callipyge phenotype and cooking method on tenderness of several major lamb muscles. Journal Animal Science, Champaign, n. 75, p. 21002105, 1997.

[31] SIMMONS, N.J.; GILBERT, K.V.; CAIRNEY, J.M. The Effect of Low Voltage Stimulation on $\mathrm{pH}$ fall and Meat Tenderness in Lambs. 43th ICoMST v. único, n. 43, p. 610-611, 1997.

[32] SINNETT-SMITH, P.A; WOOLLIAMS, J.A.; WARRIS, P.D.; ENSER, M. Effects of recombinant DNA-derived bovine somatotropin on growth, carcass characteristics and meat quality in lambs from three breeds. Anim. Prod.. Hamilton, v. 49, p. 281-289, 1989.

[33] VELASCO, S.; LAUZURICA, S.; CAÑEQUE, V.; PEREZ, C.; HUIDOBRO, F.; MANZANARES, C.; DIAZ, M.T. Carcass and Meat Quality of Talaverana Breed Sucking Lambs in Relation to Gender and Slaughter Weight. Animal Science. Hamilton, n. 70, p. 253-263, apr., 2000

[34] VERGARA, H.; MOLINA, A.; GALLEGO, L. Influence of Sex and Slaughter Weight on Carcass an Meat Quality in Light and Medium Weight Lambs Produced in Intensive Systems Meat Science. Essex, v. 52, n. 2, p. 221 226, june, 1999.

[35]WARRIS, P.D. Ciencia de la carne. Zaragoza: Acribia, 2003. 309p. 